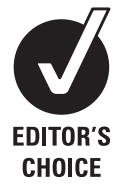

Department of Ophthalmology, Sapienza University, Umberto I Hospital, Rome, Italy

\section{Correspondence to}

Dr Magda Gharbiya, Department of Ophthalmology, Sapienza University - Umberto I Hospital, 155 Viale del Policlinico, 00161 Rome, Italy; magda.gharbiya@tiscali.it

Accepted 13 May 2012 Published Online First 2 June 2012

\title{
Long-term results of intravitreal bevacizumab for choroidal neovascularisation in pathological myopia
}

\author{
Magda Gharbiya, Filippo Cruciani, Francesco Parisi, Giovanni Cuozzo, Simona Altimari, \\ Solmaz Abdolrahimzadeh
}

\section{ABSTRACT}

Aim To evaluate the long-term results and prognostic factors of intravitreal bevacizumab (IVB) for myopic choroidal neovascularisation (mCNV).

Methods Thirty-two eyes of 30 patients with mCNV were included in a prospective case series. Treatment consisted of three monthly $1.25 \mathrm{mg}$ IVB injections. Best corrected visual acuity (BCVA) and CNV area were compared before and after treatment. Prognostic factors included in the regression analyses were age, axial length, baseline BCVA, pretreatment CNV area, CNV location and peripapillary atrophy area.

Results Results were evaluated at 2 years for 32 eyes and at 3 years for 27 eyes. Mean ( \pm SD) baseline BCVA had improved significantly from $30.1( \pm 15.6)$ letters to $45.4( \pm 13.0)$ letters at 3 years $(p<0.0001)$, with a better outcome in eyes with juxtafoveal CNV (40.4 \pm $\mathbf{1 3 . 5}$ vs. $\mathbf{5 4 . 0} \pm \mathbf{5 . 8}, p=0.001)$. Baseline BCVA correlated positively with final BCVA $(\boldsymbol{\beta}=\mathbf{0 . 5 6 0}$, $p=0.001$ ), while age showed a negative correlation $(\boldsymbol{\beta}=\mathbf{- 0 . 3 9 9}, \mathrm{p}=0.01)$. CNV area decreased from 0.63 $( \pm 0.71) \mathrm{mm}^{2}$ at baseline to $0.40( \pm 0.57) \mathrm{mm}^{2}$ at 3 years $(p<0.0001)$. Peripapillary atrophy area was the only significant contributing determinant for re-treatment (OR 1.20, 95\% Cl 1.01 to $1.42, p=0.04$ ).

Conclusions A regimen of three monthly IVB injections yielded effective and sustained results in the treatment of mCNV at 3 years of follow-up. Initial BCVA and age were the factors that correlated independently with BCVA outcome.

\section{INTRODUCTION}

Over recent years, intravitreal injection of antivascular endothelial growths factors (anti-VEGFs) has become a valid treatment option for the management of myopic choroidal neovascularisation (mCNV). It has been demonstrated that antiVEGF injection is more effective for $\mathrm{mCNV}$ than photodynamic therapy (PDT) with verteporfin. ${ }^{1-3}$ Both ranibizumab and bevacizumab have produced favourable short-term outcomes in $\mathrm{mCNV}$ without any serious ocular or systemic complications. ${ }^{4-9}$ However, in a few recent studies on the 2-year visual outcome of anti-VEGFs, the results are conflicting: visual improvement was maintained in some studies, ${ }^{10-13}$ while others reported a decline. ${ }^{14-16}$ However, the majority of these studies were retrospective and the sample size relatively small. Furthermore, there were differences in the baseline characteristics of the patients (CNV duration and site, baseline best corrected visual acuity (BCVA), previous treat ment, patients' age) and the treatment regimen.
The purpose of this prospective study was to evaluate the long-term outcomes of intravitreal bevacizumab (IVB) treatment for $\mathrm{mCNV}$. The factors predictive of both visual and anatomical outcome and the need for re-treatment were also investigated.

\section{METHODS}

This was a prospective, interventional study on 32 eyes of 30 consecutive Caucasian patients with $\mathrm{mCNV}$ who were treated with IVB between March 2006 and October 2008. The findings in some of these patients have previously been published. ${ }^{71}$ The study protocol was approved by the local ethics committee of the Sapienza University of Rome and adhered to the tenets of the Declaration of Helsinki. All participants gave written informed consent. Patients were also reminded of the off-label use of IVB.

Inclusion criteria were: (1) pathological myopia (PM) defined as a spherical equivalent >-6.0 diopters or axial length (IOLMaster, version 4.07; Carl Zeiss Meditec, Dublin, California, USA) $>26.5 \mathrm{~mm}$; (2) subfoveal or juxtafoveal CNV (CNV was classified as juxtafoveal if the lesion was $<200$ $\mu \mathrm{m}$ but not under the geometric centre of the foveal avascular zone); and (3) evidence of leakage from $\mathrm{CNV}$ on fluorescein angiography (FA). Patients were excluded if they had: (1) prior treatment for $\mathrm{CNV}$; (2) any ocular disease that could affect BCVA; (3) a history of intraocular surgery except for phacoemulsification performed within the preceding 6 months; (4) pregnancy; and (5) any systemic condition contraindicating the use of anti-VEGFs.

Before and after treatment, all patients were given a complete ophthalmic examination including BCVA measurement, optical coherence tomography (OCT), fundus photography, and digital FA. BCVA was measured using the Early Treatment Diabetic Retinopathy Study (ETDRS) chart at $4 \mathrm{~m}$ distance. OCTwas performed with the high-resolution six-radial line protocol centred on the fovea using the Stratus OCT (version 4.01; Carl Zeiss Meditec) or the Spectralis OCT (version 5.1.3.0; Heidelberg Engineering, Heidelberg, Germany) to evaluate the presence of intraretinal or subretinal fluid. Leakage from the CNV was evaluated on FA (TRC 50-LX; Topcon, Tokyo, Japan). The leakage was compared before and after treatment and was described as absent (CNV closure) or persistent. Recurrence was defined as evidence of leakage from a previously closed CNV. The area of CNV and peripapillary choroidal atrophy was measured in the early-phase FA images (within $1 \mathrm{~min}$ of dye injection), using the embedded software of the ImageNet 2000. 
Table 1 Baseline characteristics of 30 patients (32 eyes) treated with intravitreal bevacizumab

\begin{tabular}{llccc}
\hline Characteristic & Total (32 eyes) & $\begin{array}{l}\text { Subfoveal } \\
\text { (18 eyes) }\end{array}$ & $\begin{array}{l}\text { Juxtafoveal } \\
\text { (14 eyes) }\end{array}$ & p Value \\
\hline Gender (M/F) & $13 / 17$ & & & \\
Age (years) & $56.2 \pm 12.5$ & $56.3 \pm 14.7$ & $55.9 \pm 9.5$ & $0.9^{*}$ \\
Axial length (mm) & $29.9 \pm 2.3$ & $30.5 \pm 2.7$ & $29.2 \pm 1.4$ & $0.1^{*}$ \\
Baseline BCVA (No of ETDRS letters) & $30.1 \pm 15.6$ & $21.4 \pm 10.3$ & $41.3 \pm 14.2$ & $<0.0001^{*}$ \\
Baseline CNV area (mm ${ }^{2}$ ) & $0.63 \pm 0.71$ & $0.88 \pm 0.84$ & $0.28 \pm 0.16$ & $0.008^{*}$ \\
Peripapillary atrophy area (mm ${ }^{2}$ ) & $13.6 \pm 8.8$ & $15.0 \pm 10.5$ & $11.7 \pm 5.4$ & $0.3^{*}$ \\
Duration of symptoms (months) & $1.5 \pm 1.5$ & $2.0 \pm 1.8$ & $0.9 \pm 0.6$ & $0.02^{*}$ \\
Phakic/pseudophakic & $24 / 8$ & $12 / 6$ & $12 / 2$ & $0.4 \dagger$ \\
\hline
\end{tabular}

Values are mean $\pm S D$ unless otherwise indicated.

*Unpaired $t$ test with Levene's test for equality of variances.

†Fisher's exact test.

BCVA, best corrected visual acuity; CNV, choroidal neovascularisation; ETDRS, Early Treatment Diabetic Retinopathy Study.

Patients were scheduled for a loading dose of three monthly IVB injections $(1.25 \mathrm{mg} / 0.05 \mathrm{ml})$ according to the standard protocol described in our previous article. ${ }^{7}$ Re-treatment with a single bevacizumab injection was performed according to any of the following criteria: (1) evidence of persistent or recurrent leakage on FA; (2) persistent or recurrent intraretinal or subretinal fluid on OCT; (3) new subretinal haemorrhage from the mCNV. Monthly additional injections were performed until absence of fluorescein leakage from the CNV and absence of any fluid collections on OCT were obtained.

Follow-up examinations were scheduled monthly during the first 2 years and every 3 months thereafter. FA was scheduled every 3 months during the first year and every 6 months thereafter. Additional FA was performed whenever a recurrence or persistence of CNV was suspected.

\section{Statistical analysis}

Continuous variables were compared using the paired or unpaired t test as appropriate. Levene's test was used to verify variance homogeneity. Categorical variables were compared using Fisher's exact test. Forward stepwise linear regression analysis was performed to investigate the pretreatment factors predictive of BCVA outcome and CNV area after treatment. Forward stepwise logistic regression analysis was performed to evaluate the contribution of each pretreatment factor to the need for additional injections. The potential prognostic factors included in these analyses were age (years), axial length ( $\mathrm{mm}$ ), baseline BCVA (number of ETDRS letters), pretreatment CNV area $\left(\mathrm{mm}^{2}\right)$, pretreatment CNV location (subfoveal/juxtafoveal) and peripapillary atrophy area $\left(\mathrm{mm}^{2}\right)$. p Values $<0.05$ were considered significant.

\section{RESULTS}

The 2-year follow-up was completed for 32 eyes of 30 patients, and the 3-year follow-up for 27 eyes of 26 patients. Four patients (five eyes) dropped out after the second year: two patients cited personal reasons and two had difficulty in reaching the hospital because of the distance. Baseline characteristics of the patients are summarised in table 1.

All CNVs were classic on FA. Mean baseline BCVA in the 18 eyes with subfoveal CNV was significantly worse than in the 14 eyes with juxtafoveal CNV $(p<0.0001)$. Eyes with subfoveal CNV had significantly greater CNV $(p=0.008)$ and longer duration of symptoms $(p=0.02)$.

Compared with baseline, BCVA had improved significantly at all time points $(p<0.0001)$. Mean $( \pm S D)$ baseline BCVA was 30.1 ( \pm 15.6$)$ ETDRS letters. After treatment, it was 46.6 $( \pm 12.4)$ letters at 2 years (32 eyes) and 45.4 ( \pm 13.0$)$ letters at 3 years (27 eyes). Although, the greatest improvement in BCVA was seen within the first 3 months $(p<0.0001)$, visual acuity continued to improve significantly until 12 months $(p<0.0001)$ and stabilised thereafter $(p>0.05)$. Visual results over time are shown in table 2 .

Forward stepwise linear regression analysis showed that, among the pretreatment variables, initial BCVA and age were the factors that independently correlated with BCVA outcome (table 3). For every 1-letter increase in baseline BCVA, there was a mean increase in final BCVA of 0.51 letters, while for each 1 -year increase in baseline patient age, there was a mean decrease in final BCVA of 0.39 letters.

At 2 years, 23 eyes (71.9\%) had gained at least 10 letters and 20 eyes $(62.5 \%)$ had gained 15 letters or more. One $(3.1 \%)$ eye had lost 15 letters. At 3 years, 21 eyes (77.8\%) had gained at least

Table 2 Visual and anatomical outcome after intravitreal bevacizumab injection for myopic CNV

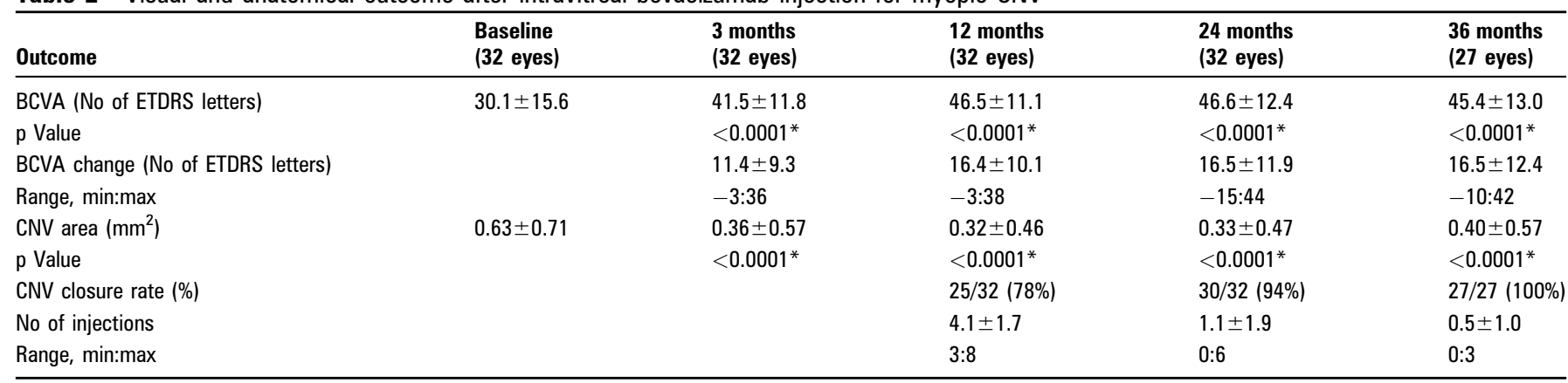

Values are mean $\pm S D$ unless otherwise indicated.

*Student $t$ test for paired data.

BCVA, best corrected visual acuity; CNV, choroidal neovascularisation; ETDRS, Early Treatment Diabetic Retinopathy Study. 
Table 3 Multivariate regression analysis to assess the influence of each pretreatment factor on BCVA at 2 and 3 years after intravitreal bevacizumab treatment

\begin{tabular}{|c|c|c|c|c|c|c|c|c|}
\hline \multirow[b]{2}{*}{ Factor } & \multicolumn{4}{|c|}{ BCVA at 2 years } & \multicolumn{4}{|c|}{ BCVA at 3 years } \\
\hline & B & $95 \% \mathrm{Cl}$ & $\beta$ & p Value & B & $95 \% \mathrm{Cl}$ & $\beta$ & p Value \\
\hline $\begin{array}{l}\text { Baseline BCVA } \\
\text { (No of ETDRS letters) }\end{array}$ & 0.51 & 0.3 to 0.7 & 0.630 & $<0.0001$ & 0.51 & 0.2 to 0.8 & 0.560 & 0.001 \\
\hline Axial length (mm) & & & -0.044 & 0.745 & & & -0.221 & 0.125 \\
\hline CNV location & & & 0.090 & 0.640 & & & 0.234 & 0.231 \\
\hline Baseline CNV area $\left(\mathrm{mm}^{2}\right)$ & & & -0.021 & 0.883 & & & -0.129 & 0.405 \\
\hline
\end{tabular}

$\beta$, standardised regression coefficient; adjusted $\mathrm{R}^{2}$, coefficient of multiple determination; $\mathrm{B}$, non-standardised regression coefficient.

BCVA, best corrected visual acuity; CNV, choroidal neovascularisation; ETDRS, Early Treatment Diabetic Retinopathy Study.

10 letters and 17 eyes (63\%) had gained 15 letters or more. Two eyes $(7.4 \%)$ had lost 10 letters. Mean changes in BCVA recorded at 2 and 3 years after treatment were $16.5( \pm 11.9)$ letters and 16.5 ( \pm 12.4$)$ letters, respectively. Forward stepwise linear regression analysis showed that baseline BCVA and age were significant contributing determinants to BCVA change after treatment (table 4). For every 1-letter increase in baseline BCVA, there was a mean decrease in BCVA of 0.49 letters, while for each 1-year increase in baseline patient age, there was a mean decrease in BCVA of 0.39 letters.

FA showed dye leakage in seven eyes $(21.9 \%)$ at 1 year (32 eyes), in two eyes (6.3\%) at 2 years (32 eyes), and in 0 eyes at 3 years (27 eyes). Mean CNV area decreased from $0.63( \pm 0.71)$ $\mathrm{mm}^{2}$ at baseline to $0.33( \pm 0.47) \mathrm{mm}^{2}$ at 2 years $(\mathrm{p}<0.0001)$ and $0.40( \pm 0.57) \mathrm{mm}^{2}$ at 3 years $(\mathrm{p}<0.0001)$. The greatest reduction in CNV area occurred within the first 3 months $(p<0.0001)$ and stabilised thereafter $(p>0.05)$. Forward stepwise linear regression analysis showed that pretreatment $\mathrm{CNV}$ area was the only significant variable affecting $\mathrm{CNV}$ area after IVB $(\beta=0.89$, $p<0.0001$ at 2 years and $\beta=0.91, p<0.0001$ at 3 years $)$. The adjusted $R^{2}$ of the final model was 0.789 at 2 years and 0.822 at 3 years.

The mean number of anti-VEGF injections was 4.1 ( \pm 1.7$), 1.1$ $( \pm 1.9)$ and $0.5( \pm 1.0)$ at 1,2 and 3 years, respectively. Fifteen (46.9\%) of the 32 treated eyes received only the loading dose of three IVB injections. Forward stepwise logistic regression analysis showed that peripapillary atrophy area was the only significant contributing determinant to the need for additional injections (OR 1.24, 95\% CI 1.04 to $1.48, p=0.02$ at 2 years; OR $1.20,95 \%$ CI 1.01 to $1.42, p=0.04$ at 3 years).

There were no serious adverse systemic or ocular events, such as endophthalmitis, retinal detachment, cataract or glaucoma, during the follow-up period.

\section{Subgroup analysis of subfoveal versus juxtafoveal CNV}

In eyes with subfoveal CNV, BCVA had improved from 21.4 $( \pm 10.3)$ to $41.7( \pm 13.1)$ letters at 2 years $(p<0.0001)$ and to 40.4 ( \pm 13.5$)$ letters at 3 years $(p<0.0001)$. Similarly, in eyes with juxtafoveal CNV, BCVA had improved from 41.3 ( \pm 14.2$)$ to 52.9 ( \pm 8.2$)$ letters at 2 years $(p=0.01)$ and to $54.0( \pm 5.8)$ letters at 3 years $(p=0.02)$. BCVA in juxtafoveal CNV was significantly better than in subfoveal CNV after treatment $(p=0.006$ at 2 years and $p=0.001$ at 3 years), while BCVA gain tended to be higher in subfoveal than in juxtafoveal CNV $(p=0.04$ and $p=0.2$ at 2 and 3 years, respectively). In eyes with subfoveal CNV, mean CNV area had decreased from 0.88 $( \pm 0.84)$ to $0.45( \pm 0.59) \mathrm{mm}^{2}$ at 2 years $(\mathrm{p}<0.0001)$ and to 0.54 $( \pm 0.68) \mathrm{mm}^{2}$ at 3 years $(\mathrm{p}<0.0001)$. Similarly, in eyes with juxtafoveal lesions, mean $\mathrm{CNV}$ area had decreased from 0.28 $( \pm 0.16)$ to $0.16( \pm 0.13) \mathrm{mm}^{2}$ at 2 years $(p=0.002)$ and to 0.18 $( \pm 0.15) \mathrm{mm}^{2}$ at 3 years $(\mathrm{p}=0.03)$. Although the difference was marginally significant, the post-treatment mean $\mathrm{CNV}$ area in juxtafoveal CNV tended to be smaller than in subfoveal CNV $(p=0.05$ at 2 years and $p=0.05$ at 3 years). No significant difference was found in the mean number of injections between subfoveal and juxtafoveal CNV ( $p=0.4$ and $p=0.9$ at 2 and 3 years, respectively). Results of subgroup analysis are shown in table 5 .

\section{DISCUSSION}

In this prospective study, our results showed that IVB therapy significantly improved BCVA in mCNV for up to 3 years of follow-up. Pretreatment BCVA was the most important prognostic factor positively affecting long-term BCVA. Thus patients with better BCVA at baseline also had better BCVA after treatment. In addition, regression analysis showed that baseline

Table 4 Multivariate regression analysis to assess the influence of each pretreatment factor on BCVA change at 2 and 3 years after intravitreal bevacizumab treatment

\begin{tabular}{|c|c|c|c|c|c|c|c|c|}
\hline \multirow[b]{2}{*}{ Factor } & \multicolumn{4}{|c|}{ BCVA change at 2 years } & \multicolumn{4}{|c|}{ BCVA change at 3 years } \\
\hline & $\bar{B}$ & $95 \% \mathrm{Cl}$ & $\beta$ & p Value & $\bar{B}$ & $95 \% \mathrm{Cl}$ & $\beta$ & p Value \\
\hline $\begin{array}{l}\text { Baseline BCVA } \\
\text { (No of ETDRS letters) }\end{array}$ & -0.49 & -0.7 to -0.3 & -0.632 & $<0.0001$ & -0.49 & -0.8 to -0.2 & -0.572 & 0.001 \\
\hline Age (years) & -0.32 & -0.6 to -0.06 & -0.338 & 0.019 & -0.39 & -0.7 to -0.1 & -0.417 & 0.01 \\
\hline CNV location & & & 0.094 & 0.640 & & & 0.244 & 0.231 \\
\hline Axial length (mm) & & & -0.045 & 0.745 & & & -0.231 & 0.125 \\
\hline Baseline CNV area $\left(\mathrm{mm}^{2}\right)$ & & & -0.022 & 0.883 & & & -0.135 & 0.405 \\
\hline
\end{tabular}

$\beta$, standardised regression coefficient; adjusted $R^{2}$, coefficient of multiple determination; $B$, non-standardised regression coefficient.

BCVA, best corrected visual acuity; CNV, choroidal neovascularisation; ETDRS, Early Treatment Diabetic Retinopathy Study. 
Table 5 Subfoveal versus juxtafoveal myopic CNV: visual and anatomical outcome after intravitreal bevacizumab injection

\begin{tabular}{|c|c|c|c|c|c|c|c|c|}
\hline \multirow[b]{2}{*}{ Outcome } & \multicolumn{2}{|l|}{ Baseline } & \multicolumn{2}{|l|}{1 year } & \multicolumn{2}{|l|}{2 years } & \multicolumn{2}{|l|}{3 years } \\
\hline & $\begin{array}{l}\text { Subfoveal } \\
\text { (18 eyes) }\end{array}$ & $\begin{array}{l}\text { Juxtafoveal } \\
\text { (14 eyes) }\end{array}$ & $\begin{array}{l}\text { Subfoveal } \\
\text { (18 eyes) }\end{array}$ & $\begin{array}{l}\text { Juxtafoveal } \\
\text { (14 eyes) }\end{array}$ & $\begin{array}{l}\text { Subfoveal } \\
\text { (18 eyes) }\end{array}$ & $\begin{array}{l}\text { Juxtafoveal } \\
\text { (14 eyes) }\end{array}$ & $\begin{array}{l}\text { Subfoveal } \\
\text { (17 eyes) }\end{array}$ & $\begin{array}{l}\text { Juxtafoveal } \\
\text { (10 eyes) }\end{array}$ \\
\hline $\begin{array}{l}\text { BCVA } \\
\text { (No of ETDRS letters) }\end{array}$ & $21.4 \pm 10.3$ & $41.3 \pm 14.2$ & $42.2 \pm 11.9$ & $52.1 \pm 6.9$ & $41.7 \pm 13.1$ & $52.9 \pm 8.2$ & $40.4 \pm 13.5$ & $54.0 \pm 5.8$ \\
\hline $\mathrm{p}$ Value & & $<0.0001^{*}$ & & $0.006^{*}$ & & $0.006^{*}$ & & $0.001^{*}$ \\
\hline $\begin{array}{l}\text { BCVA change } \\
\text { (No of ETDRS letters) }\end{array}$ & & & $20.8 \pm 7.2$ & $10.9 \pm 10.9$ & $20.3 \pm 8.1$ & $11.6 \pm 14.4$ & $18.7 \pm 10.7$ & $12.8 \pm 14.8$ \\
\hline $\mathrm{p}$ Value & & $0.008^{*}$ & & $0.07^{*}$ & & $0.05^{*}$ & & $0.05^{*}$ \\
\hline CNV closure rate $(\%)$ & & & 13/18 (72\%) & $12 / 14(86 \%)$ & $18 / 18(100 \%)$ & $12 / 14(86 \%)$ & $17 / 17(100 \%)$ & $10 / 10(100 \%)$ \\
\hline $\mathrm{p}$ Value & & & & $0.4 \dagger$ & & $0.2 \dagger$ & & - \\
\hline No of injections & & & $4.4 \pm 1.8$ & $3.7 \pm 1.5$ & $1.3 \pm 2.0$ & $0.7 \pm 1.8$ & $0.5 \pm 1.0$ & $0.5 \pm 1.1$ \\
\hline $\mathrm{p}$ Value & & & & $0.3^{*}$ & & $0.4^{*}$ & & $0.9^{*}$ \\
\hline
\end{tabular}

Values are mean $\pm S D$ unless otherwise indicated.

*Unpaired $t$ test with Levene's test for equality of variances.

†Fisher's exact test.

BCVA, best corrected visual acuity; CNV, choroidal neovascularisation; ETDRS, Early Treatment Diabetic Retinopathy Study.

BCVA correlated negatively with BCVA change after treatment. Therefore patients with higher BCVA at baseline had less BCVA gain after treatment. This may be due to the so-called 'ceiling-floor effect' - that is, patients with higher pre-treatment BCVA have a smaller chance of improvement. This should be kept in mind so that IVB is not considered less effective in patients with better baseline BCVA. Overall, the visual results of the current series are consistent with those of a retrospective study by Nakanishi et al, who reported significant BCVA improvement which was sustained for 2 years in 23 treatmentnaïve eyes with $\mathrm{mCNV}$ treated with IVB and, as in our evaluation of visual results, took into consideration the ceiling-floor effect. $^{13}$

In the literature, the long-term outcomes of IVB in the treatment of $\mathrm{mCNV}$ are conflicting, and there are some studies that report a decline of visual improvement during the second year after treatment. Ruiz-Moreno et al prospectively examined the 2-year results of 19 eyes with $\mathrm{mCNV}$ treated with three loading IVB injections. BCVA was significantly improved at 1 year but the significance of the improvement was not maintained at 2 years. However, eight (42\%) of the 19 treated eyes had received previous PDT before IVB treatment, and the subgroup analysis showed a better visual outcome in treatmentnaïve eyes. ${ }^{15}$ Indeed, earlier studies reported that previous PDT may worsen the prognosis of mCNV treated by IVB. ${ }^{4} 17$ This may explain the increased efficacy of IVB therapy obtained in our study where treatment-naïve eyes only were investigated. Ikuno et al in a retrospective case series reported the 2-year results of 11 treatment-naïve eyes after IVB injection for mCNV. BCVA was significantly improved at 1 year, but the significance was not maintained at 2 years. However, this study examined only women with a mean age of $67.8 \pm 6.2$ years. ${ }^{14}$ It is known that, without treatment, older patients with mCNV have a worse visual prognosis than younger patients. ${ }^{18} 19$ It has also been demonstrated that older patients with $\mathrm{mCNV}$ have worse visual outcome than younger patients after PDT. ${ }^{20}$ This suggests that the results of Ikuno et al may have been influenced by the older age of the recruited patients. In our results, the regression analysis showed that age at onset was a negative prognostic factor significantly affecting both BCVA outcome and BCVA change after treatment.

In our study, BCVA had significantly improved after treatment in both subfoveal and juxtafoveal $\mathrm{CNV}$. In a recent prospective study, Hayashi et al reported a significant BCVA improvement for up to 2 years in 75 treatment-naïve eyes after IVB injection. However, in contrast with our findings, subgroup analysis revealed that BCVA improved significantly only in non-subfoveal CNV, while there was no significant improvement in subfoveal lesions. ${ }^{21}$ The design of the study by Hayashi et al was based on a single dose of IVB administered at baseline followed by pro re nata treatment, whereas the design of our study was based on a treatment regimen of three loading doses of bevacizumab followed by pro re nata treatment. This suggests that a more aggressive initial approach may improve visual prognosis even in subfoveal mCNV. Indeed, RuizMoreno et al, prospectively comparing the two treatment regimens (three loading doses vs a single dose of bevacizumab) in 39 eyes with $\mathrm{mCNV}$, showed a significantly higher recurrence rate in the group treated with a single IVB injection. ${ }^{22}$ mCNV may show latent activity, which is not always easily detected. Angiographic leakage is sometimes masked by deep atrophy and/or pigment clumping, and even the more recent Fourier-domain OCTs may not be accurate enough to detect subtle signs. Thus we may be undertreating this condition. An induction phase consisting of three monthly injections may increase the chances of complete inactivation of $\mathrm{CNV}$, thus improving visual prognosis.

Our anatomical results showed an absence of leakage from $\mathrm{CNV}$ in all eyes at 3 years. IVB injection induced a significant decrease in CNV area. In a comparative study, Hayashi et al demonstrated that CNV closure in IVB-treated eyes was accompanied by significant shrinkage of CNV, whereas the CNV area did not decrease, or even increased, in PDT-treated eyes. ${ }^{1}$ This shrinkage may, to some extent, explain the better visual prognosis of IVB-treated eyes.

Finally, we evaluated the prognostic factors associated with the need for re-treatment, and peripapillary atrophy area was the most important factor. This is intriguing. Yasuzumi et al evaluated peripapillary crescent enlargement in PM. They concluded that progression of choroidal circulatory disturbances may contribute to crescent enlargement in these eyes. ${ }^{23}$ Thus it could be speculated that peripapillary atrophy size may be related to the degree of choroidal ischaemia in PM. Choroidal ischaemia is known to induce growth factor release (namely, VEGF), which in turn may increase CNV activity and decrease CNV response to IVB treatment. 
The limitations of the present study are the small sample size and the lack of a control group. The strengths include the prospective design, an extended follow-up, and the high rate of adherence to a strict protocol.

In conclusion, our results showed that BCVA improvement was maintained for up to 3 years after IVB treatment in eyes with subfoveal and juxtafoveal CNV. In addition we found that the most important prognostic factors of visual outcome were, in decreasing order of impact, baseline BVCA and age.

Contributors Each author certifies that they have made substantial contribution to the work reported in this manuscript by participating in at least the following three areas: (1) substantial contributions to conception and design, acquisition of data, or analysis and interpretation of data; (2) drafting the article or revising it critically for important intellectual content; and (3) final approval of the version to be published.

Competing interests None.

Patient consent Obtained.

Ethics approval Provided by the local ethics committee of the Sapienza University of Rome.

Provenance and peer review Not commissioned; externally peer reviewed.

\section{REFERENCES}

1. Hayashi K, Ohno-Matsui K, Teramukai S, et al. Comparison of visual outcome and regression pattern of myopic choroidal neovascularization after intravitreal bevacizumab or after photodynamic therapy. Am J Ophthalmol 2009;148:396-408.

2. Yoon JU, Byun YJ, Koh HJ. Intravitreal anti-VEGF versus photodynamic therapy with verteporfin for treatment of myopic choroidal neovascularization. Retina 2010:30:418-24.

3. El Matri L, Kort F, Chebil A, et al. Intravitreal bevacizumab versus photodynamic therapy for myopic choroidal neovascularisation in a North-African population. Graefes Arch Clin Exp Ophthalmol 2011;249:1287-93.

4. Chan WM, Lai TY, Liu DT, et al. Intravitreal bevacizumab (Avastin) for myopic choroidal neovascularisation: 1-year results of a prospective pilot study. $\mathrm{Br} \mathrm{J}$ Ophthalmol 2009;93:150-4.

5. Ruiz-Moreno JM, Montero JA, Gomez-Ulla F, et al. Intravitreal bevacizumab to treat subfoveal choroidal neovascularisation in highly myopic eyes: 1-year outcome. Br J Ophthalmol 2009;93:448-51.

6. Ikuno Y, Sayanagi K, Soga K, et al. Intravitreal bevacizumab for choroidal neovascularization attributable to pathological myopia: one-year results. $A m \mathrm{~J}$ Ophthalmol 2009;147:94-100

7. Gharbiya M, Allievi F, Mazzeo L, et al. Intravitreal bevacizumab treatment for choroidal neovascularization in pathologic myopia: 12-month results. Am J Ophthalmol 2009;147:84-93.
8. Lai TY, Chan WM, Liu DT, et al. Intravitreal ranibizumab for the primary treatment of choroidal neovascularization secondary to pathologic myopia. Retina 2009:29:750-6.

9. Vadalà $\mathbf{M}$, Pece $\mathrm{A}$, Cipolla $\mathrm{S}$, et al. Is ranibizumab effective in stopping the loss of vision for choroidal neovascularisation in pathologic myopia? A long-term follow-up study. Br J Ophthalmol 2011;95:657-61.

10. Baba T, Kubota-Taniai M, Kitahashi M, et al. Two-year comparison of photodynamic therapy and intravitreal bevacizumab for treatment of myopic choroidal neovascularisation. $\mathrm{Br} \mathrm{J}$ Ophthalmol 2010:94:864-70.

11. Gharbiya M, Allievi F, Conflitti S, et al. Intravitreal bevacizumab for treatment of myopic choroidal neovascularization: the second year of a prospective study. Clin Ter 2010;161:e87-93.

12. Chen $\mathbf{C H}$, Wu PC, Chen YJ, et al. Intravitreal injection of $2.5 \mathrm{mg}$ bevacizumab for treatment of myopic choroidal neovascularization in treatment-naive cases: a 2-year follow-up. J Ocul Pharmacol Ther 2011;27:395-400.

13. Nakanishi H, Tsujikawa A, Yodoi Y, et al. Prognostic factors for visual outcomes 2 years after intravitreal bevacizumab for myopic choroidal neovascularization. Eye 2011:25:375-81

14. Ikuno Y, Nagai Y, Matsuda S, et al. Two-year visual results for older Asian women treated with photodynamic therapy or bevacizumab for myopic choroidal neovascularization. Am J Ophthalmol 2010;149:140-6.

15. Ruiz-Moreno JM, Montero JA. Intravitreal bevacizumab to treat myopic choroidal neovascularization: 2-year outcome. Graefes Arch Clin Exp Ophthalmol 2010;248:937-41.

16. Voykov B, Gelisken F, Inhoffen W, et al. Bevacizumab for choroidal neovascularization secondary to pathologic myopia: Is there a decline of the treatment efficacy after 2 years? Graefes Arch Clin Exp Ophthalmol 2010:248:543-50.

17. Ruiz-Moreno JM, Montero JA, Gomez-Ulla F. Photodynamic therapy may worsen the prognosis of highly myopic choroidal neovascularisation treated by intravitreal bevacizumab. Br J Ophthalmol 2009;93:1693-4.

18. Kojima A, Ohno-Matsui K, Teramukai S, et al. Estimation of visual outcome without treatment in patients with subfoveal choroidal neovascularization in pathologic myopia. Graefes Arch Clin Exp Ophthalmol 2006:244:1474-9.

19. Yoshida T, Ohno-Matsui $K$, Ohtake $Y$, et al. Longterm visual prognosis of choroida neovascularization in high myopia: a comparison between age groups. Ophthalmology 2002;109:712-19.

20. Axer-Siegel R, Ehrlich R, Weinberger D, et al. Photodynamic therapy of subfoveal choroidal neovascularization in high myopia in a clinical setting: visual outcome in relation to age at treatment. Am J Ophthalmol 2004;138:602-7.

21. Hayashi K, Shimada N, Moriyama M, et al. Two-year outcomes of intravitreal bevacizumab for choroidal neovascularization in Japanese patients with pathologic myopia. Retina 2012;32:687-95.

22. Ruiz-Moreno JM, Montero JA, Amat-Peral P. Myopic choroidal neovascularization treated by intravitreal bevacizumab: comparison of two different initial doses. Graefes Arch Clin Exp Ophthalmol 2011;249:595-9.

23. Yasuzumi K, Ohno-Matsui K, Yoshida T, et al. Peripapillary crescent enlargement in highly myopic eyes evaluated by fluorescein and indocyanine green angiography. $\mathrm{Br} \mathrm{J}$ Ophthalmol 2003;87:1088-90. 